\title{
ROI-based features for classification of skin diseases using a multi-layer neural network
}

\author{
Thanh-Hai Nguyen, Ba-Viet Ngo
}

Ho Chi Minh City University of Technology and Education, Vietnam

\begin{tabular}{l} 
Article Info \\
\hline Article history: \\
Received Feb 17, 2021 \\
Revised Apr 14, 2021 \\
Accepted Apr 20, 2021 \\
\hline
\end{tabular}

\section{Keywords:}

GLCM algorithm

Matrix confusion

MLNN structure

ROI features

Skin diseases

\begin{abstract}
Skin diseases have a serious impact on human life and health. This article aims to represent the classification accuracy of skin diseases for supporting the physicians' correct decision on patients for early treatment. In particular, 100 images in each type of five skin diseases from ISIC database are used for balanced datasets related to the classification accuracy. In addition, this paper focuses on processing images for extracting six optimal types of eleven features of skin disease image for higher classification performance and also this takes less time for training. Therefore, skin disease images are filtered and segmented for separating region of interests (ROIs) before extracting optimal features. First, the skin disease images are processed by normalizing sizes, removing noises, segmenting to separate region of interests (ROIs) showing skin disease signs. Next, a gray-level co-occurrence matrix (GLCM) method is applied for texture analysis to extract eleven features. With the optimal six features chosen, the high classification accuracy of skin diseases is about $92 \%$ evaluated using a matrix confusion. The result showed to illustrate the effectiveness of the proposed method. Furthermore, this method can be developed for other medical datasets for supporting in disease diagnosis.
\end{abstract}

This is an open access article under the CC BY-SA license.

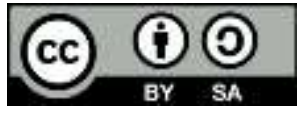

\section{Corresponding Author:}

Thanh-Hai Nguyen

Faculty of Electrical and Electronics Engineering

Ho Chi Minh City University of Technology and Education

01 Vo Van Ngan Street, Linh Chieu Ward, Thu Duc City, Ho Chi Minh City, Viet Nam

Email: nthai@hcmute.edu.vn

\section{INTRODUCTION}

Skin disease, which is one of the most common diseases in humans, can affect to all ages and sexes. In current, there are many patients with different skin diseases and a lot of deaths worldwide. Moreover, the skin disease is a global problem, ranking 18th in the global ranking of worldwide health burdens. In a 2017 global statistic, skin diseases were about $1.79 \%$ of other diseases [1], in which the skin diseases include dermatitis (allergies, contact, sebum), acne, and urticaria, psoriasis, viral skin disease, fungal skin disease, scabies, melanoma, pyodermatitis, cellulitis, carcinoma, ulcerative by cancer.

In recent years, enhancement of medical images before segmentation for image recognition or classification have been applied [2]. In skin disease identification, all skin disease images were segmented using enhanced level [3], [4]. A gray level co-occurrence matrix (GLCM) were applied to these segmented images for extracting features applied to a dragonfly-based deep neural networks (DNN) classifier. It is obvious that a medical image is often filtered noise for segmenting region of interest (ROI), in which enhancement of the image for increasing classification accuracy is very important. 
Images are captured from different sources and always exist different noises. In practice, there are types of filters for removing noises in image. In order to eliminate unwanted noise in an image and sharpen it, the combined filter of Gaussian and Butterworth high pass was applied [5], [6] in the frequency domain. In [7]-[9], images of skin lesions were segmentation using a growing method, in which automatic initialization of seed points were used. The result of segementation to extract lesion areas in images was used in the fusion of a support vector machine (SVM) and k-neural network (k-NN) for classification with $61 \%$ of F-measure. In our research, the Butterworth high pass filter will be employed to sharpen areas of skin disease before segmentation.

In image processing, image segmentation algorithm [10] is commonly applied to separate object from an image for analysis or recognition, Otsu method is one of thresholding methods are often employed for segmentation thresholds [11]. Furthermore, the Otsu segmentation method is utilized to determine threshold for disease region detection for classification [12]. In one application, authors applied to find key points of pixels considered as the seed points of diseased skin. Therefore, features corresponding to the key points extracted after the segmentation were applied to be input of the classifier [13]. In our article, the Otsu method is applied to segment skin lesion areas, called ROI.

In practice, there are different filter methods for enhancing images such as median and bottom hat filters which are often applied for de-noising in medical images. Authors proposed a novel approach for detecting features, in which a Bottom hat filtering was employed for smoothing images [4], [14]. Moreover, the median filter is often utilized for remove salt and pepper noise in medical images. In this research, we will apply median and bottom hat filters for eliminating noises in skin diseases and shappen skin disease areas.

For effective segmentation to separate ROI in image, edge detection of objects in the image is necessary. In [15], authors proposed a Canny edge detector with a iterative median filter (IMF) for skin lesion border detection, in which the improved iterative segmentation algorithm for border detection of real skin lesions produced the effective result. The performance of this algorithm is better than the traditional segmentation algorithm. In our paper, the Canny method is applied for detecting edges in skin disease areas in image for separating ROI.

Morphological operation [16], [17] was employed in processing T1-weighted magnetic resonance imaging (MRI) images, in which dilation is to enhance the estimation volume method. It is obvious that this method is better compared to Stereology method for less MRI slices and less test points. In deep learning methods for skin lesion classification, primary morphological elements are a module in the automatic detection system. The result of this classification was the $76.00 \%$ accuracy for 5 classes of the primary morphological elements and the $81.67 \%$ accuracy for 3 classes [18]. In our study, dilation and erosion in the morphological approach are applied for removing unwanted small objects and connecting dotted lines in skin disease areas.

In medical images, the separation of ROIs for feature extraction is very necessary. A simple and reliable approach for skin region segmentation to generate ROI can be applied. In one research, a method in terms of segmenting solid skin regions without generating much noisy segments was proposed in [19]. A ROI could be separated based on analysis of fine geometric details of skin lesion images. The result was that the average performance of this method was better the current state-of-the-art techniques without training [20]. It is obvious that there have been different methods for separating a ROI in image. In our research, after pre- processing image, a Canny edge detection will be applied to separate ROI for extracting optimal features using GLCM method.

For skin disease classification, image feature extraction using GLCM method plays an important role because it can contain important information of the affected skin. For feature extraction for texture recognition, a GLCM method was employed [21]-[24]. In this research, three different datasets were proposed, in which the first set is a simple modified features extracted from the traditional GLCM; the second set uses two sub-sets of features extracted from two GLCM methods calculated using two displacement values; the third one was passing the extracted set of the GLCM through an artificial neural network (ANN) for classification. In current, many methods have been applied for classification of skin lesions [25], [26]. Therefore, these feature components were used to be the input of the classifier to classify different skin diseases. Our study is that a GLCM is utilized for extracting features and just 6 optimal features of eleven ones used for classification of skin diseases.

With many different human skin diseases, it is very important to classify and find the disease automatically because it helps doctors to early diagnose the disease and the patient can be treated early and effectively. In recent years, the classification of different components using an artificial intelligence (AI) method has been very popular and effective [27], [28]. In particular, authors proposed a deep convolutional networks with a sustained combination for the classification of damaged skin diseases [27]. With this proposed method, the deep learning network was employed for testing over 900 skin disease images and it 
gave relatively good results. Artificial intelligence algorithms were proposed for classifying skin diseases [29], [30]. In particular, a CNN model combined intra-architecture and inter-architecture network fusion [31] or a compact deep learning-based classification model with a separable convolutional neural network [32], the state-of-art DNN [33] were utilized. In one research, authors proposed a region-based CNN (FRCNN) for training clinical images of pigmented skin lesions, in which there were malignant tumor images [34]. The results of this research was compared to another research to illustrate the better proposed method. In research [35], a CNN was applied in the classification of Psoriasis skin disease with the accuracy rate of $82.9 \%$ and $72.4 \%$ for Plaque and Guttate Psoriasis skin disease, respectively. It is obvious that classification models using AI methods for big medical image sets are very important.

In this article, we have proposed an automatic classification method, in which six optimal features are extracted from five balanced types of skin diseases and then they are combined to a multi layer neural network (MLNN) [36], [37] for high classification. All these images are preprocessed to remove unnecessary components, as well as to enhance them before segmentation for separating ROIs before extracting optimal features using a GLCM. These extracted features are used to be the inputs of the MLNN classifier. The average accuracy of the high classification is evaluated using a confusion matrix.

\section{METHODOLOGY}

It is very important to detect and classify human skin disease early because it can help the physicians' correct diagnosis for treatment early. In this research, a skin disease detection method is based on the extraction of the surface texture features of the disease skin image. Therefore, the feature set will be statistically analyzed to select the optimal features for training and classification process using a MLNN structure. It is obvious that this will take more time for processing disease skin images and selecting optimal features before skin disease classification with high accuracy. With selected optimal features, a simpler MLNN structure can be applied, in which its training takes less time and classification performance is high.

\subsection{Skin image pre-processing for determining ROI}

Image pre-processing is an important stage in the extraction of image features for classifying skin diseases. Therefore, image datasets are processed to enhance the image quality before extracting structural features for the skin disease classification with higher accuracy using neural networks. To analyze the diseased skin objects in the image, we need to distinguish the objects of interest from the rest of the image, called the background. These objects can be identified using a segmentation method to separate the foreground from the image for collecting ROI. The ROI part may contain different features such as color, uniformity, texture, gray level, frequency or moment. These features can form a feature vector associated with the ROI and this helps to distinguish different skin lesions using a neural network.

In this paper, the process of ROI separation will be carried out through seven steps. The pre-processing steps will make the better skin disease image before performing the segmentation to separate the ROI from the skin disease image for feature extraction. In particular, the steps for processing of skin images are,

Step 1: The original image $f(x, y)$ will be normalized for the same size. Then the image enhancement $g(x, y)$ is calculated by convoluting a kernel $k_{l}(s, t)$ with the image $f(x, y)$,

$$
g(x, y)=\sum_{s=-a}^{a} \sum_{t=-b}^{b} k_{1}(s, t) f(x-s, y-t)
$$

Step 2: The image after enhancement will be transformed to produce the Fourier images $G(u, v)$ in the frequency domain before filtering out the low-frequency component. In this study, the Butterworth high pass filter was applied to sharpen for the purpose of enhancing details, highlighting particles in the ROI region, which are considered the lesions of skin diseases. The image $\tilde{G}(u, v)$ using the Butterworth filter is described,

$$
\left\{\begin{array}{c}
\tilde{G}(u, v)=H(u, v) * D(u, v) \\
H(u, v)=\frac{1}{1+\left[D_{0} / D(u, v)\right]^{2 n}}
\end{array}\right.
$$

where $\mathrm{D}(u, v)$ is the Euclidean Distance from any point $(u, v)$ in the image $\mathrm{G}(u, v)$ to the origin of the frequency plane, $D(u, v)=\sqrt{u^{2}+v^{2}}$ and $D_{0}$ describes the cut-off frequency.

Step 3: The filtered image using the Butterworth filter will be converted into the image $\tilde{f}(x, y)$ in the spatial domain before using the Otsu [4] method for finding the threshold for image segmentation and for the 
conversion to binary images $\tilde{f}_{b}(x, y)$. The segmentation is to remove the background of the normal skin to be black, and keep the diseased skin to be white in the binary image.

Step 4: The binary image will be removed salt and pepper noise by the median filter $\tilde{f}_{n}(x, y)$.

Step 5: A bottom-hat filter will be applied to the image $\tilde{f}_{n}(x, y)$ after removing some elements not to be in the disease skin image area, such as hairs or others. The image $\tilde{g}(x, y)$ is calculated using the bottom-hat filter multiplied with the kernel $k_{2}(s, t)$,

$$
\tilde{g}(x, y)=\tilde{f}_{n}(x, y)+\sum_{s=-a}^{a} \sum_{t=-b}^{b} k_{2}(s, t) \tilde{f}_{n}(x-s, y-t)
$$

Step 6: The filtered image $\tilde{g}(x, y)$ is eroded to remove the unknown small areas and then detected the edge of the disease skin area using a Canny method [15]. Therefore, the image with the disease skin edge is dilated to connect the dotted lines.

Step 7: Finally, finding the region with the largest area to separate the ROI is performed. In particular, to find this largest region, it is necessary to identify the regions filled in the binary image. Therefore, the connected object components are found and each filled object is assigned a label for identification. From the labeled objects, we can determine the area of the objects based on the number of pixels and the index for extracting the object with the largest area.

The result obtained after pre-processing is the ROI area of the skin disease to serve for feature extraction. It means that pre-processing for separating the ROI area can take more time, but it will take less time for training process using the MLNN for classifying skin disease images.

\subsection{Feature extraction of ROI}

After extracting the ROI region from the skin disease image, a GLCM method is employed for feature extraction. With different skin color images, there is a difference in the skin lesion area related to structure, frequency, and other parameters. With the texture distribution and parameters in the GLCM algorithm of the skin disease image, we can obtain different features by the analysis of skin texture, roughness, uniformity of the skin, and skin condition.

The GLCM algorithm is one of methods used for extracting important features related to image texture analysis. In particular, each element in the image represents the probability of occurring the same intensity at the typical distance $d$ and the angle $\theta$. Therefore, there can be many different GLCM matrices depending on the pair of $d$ and $\theta$. In this study, with damaged skin disease, only some important features such as contrast, energy, homogeneity, mean, standard deviation, entropy, which are synchronous and repetitive, are considered. From these selected features, their vectors are used in the MLNN classifier. In the GLCM algorithm, features such as contrast, energy, homogeneity, mean, standard deviation, entropy can greatly differ from other groups and so they can be datasets chosen for training and classifying in the MLNN. After pre-processing skin image and separating the ROI of the image $G(i, j)$, the optimal following features will be calculated using the GLCM.

Contrast feature is to measure the spatial frequency of skin image which is the difference between the highest and lowest values of a contiguous/adjacent set of pixels. In particular, the contrast can measure the amount of local variations present the skin image. In addition, the contrast describes the depth of "textile grooves" of the image. Therefore, if the contrast value is higher, the "grooves" is deeper. The contrast feature cab be calculated using the following formula,

$$
C t=\sum_{i}^{L-1} \sum_{j}^{L-1}(i-j)^{2} P(i, j)
$$

in which $|\mathbf{i}-\mathbf{j}|$ is the grayscale difference between adjacent pixels, $P(\mathbf{i}, \mathbf{j})$ is the element $(\mathbf{i}, \mathbf{j})$ of the normalized symmetrical GLCM, called the distribution probability of the different grayscale levels between the adjacent pixels. $\mathrm{L}$ is the number of gray levels in the skin disease image.

Entropy is an important feature which allows to measure information of the disorder or complexity of skin image. In particular, the entropy is large when the skin image is not texturally uniform, in which the entropy is high, potentially the part of skin image has complex textures. In addition, when the entropy is strongly, it may inversely correlate to energy. The entropy value is calculated using the following formula:

$$
E t=\sum_{i}^{L-1} \sum_{j}^{L-1} P(i, j) \log P(i, j)
$$

Inverse Difference Moment is called homogeneity of skin image and can show lager values for smaller gray tone differences in pair elements. In addition, it is more sensitive to the presence of near diagonal elements in the GLCM. Thus, it has maximum value when all elements in the skin image are same. In addition, homogeneity is used to describe the roughness of the image structure. It means that if the 
homogeneity $\mathrm{Hg}$ value is greater, the roughness of the image structure at that damaged area will be greater. The uniform value of skin diseases can be described,

$$
H g=\sum_{i}^{L-1} \sum_{j}^{L-1} \frac{1}{1+(i-j)^{2}} P(i, j)
$$

With the feature based on energy, the image with lesion skin part can produce a maximum value. Moreover, this energy method is to measure the texture uniformity and detects disorders in textures and is used to describe the thickness of the structure of a skin disease image. The energy of this skin disease image can be described using the following expression,

$$
E g=\sum_{i}^{L-1} \sum_{j}^{L-1} G(i, j)^{2}
$$

Mean is the average gray level of the image of skin disease where $G(x, y)$ is the image after preprocessing with the size $m \times n$. In addition, the gray intensity of pixels is normalized in the range [0,1] before calculating the average of skin disease images,

$$
M e=\frac{\sum_{x=0}^{m-1} \sum_{y=0}^{n-1} G(x, y)}{255 \times m \times n}
$$

Feature related to calculation is standard deviation. This feature represents a comparison of the mean standard deviation values of different skin diseases. It can be seen that the difference between the disease classes can be described when analyzing the standard deviation of the gray level of the skin image,

$$
S D=\sqrt{\frac{\sum_{x=0}^{m-1} \sum_{y=0}^{n-1}\left(\frac{G(x, y)}{255}-M e\right)^{2}}{m \times n}}
$$

Analysis of the features of skin diseases is important for classification. However, there is an excess between parameters or some features without the difference between skin diseases. It is obvious that this will take time for calculation without benefit for training process of skin disease classification. In this study, the calculation and statistics of the features of the skin diseases will be the basis for the selection of features that can reflect the skin features at different levels. After analyzing datasets from eleven types of different features using the GLCM algorithm, the results showed that there are six optimal features chosen: contrast, energy, homogeneity, mean, standard deviation, entropy. These feature types show the large change between different skin diseases and are used to effectively train for the skin disease classification.

\subsection{Multilayer neural network structure for skin disease classification}

In this research, as the number of skin diseases increases, it becomes more difficult to directly classify skin diseases with high accuracy without pre-processing images. Therefore, it is necessary to preprocess the image before segmentation of separating ROIs and this will optimize training process and classification with high accuracy using the MLNN. Previous works have shown that the neural network can be well applied in medical diagnostic systems [29], [30]. Therefore, in our article, the classification of skin diseases will be performed using the MLNN, this can result in a higher accuracy classification using six optimal features of skin disease images.

The MLNN is the ability of better processing complex relationships between different parameters and then effectively classifying based on learning from the processed training data. The success of a classification system based on the MLNN depends on the model architecture of the network and the training algorithm. Furthermore, the number of hidden layers as well as the number of nodes in the network are determined using the trial and error method during the classification process repeated. In particular, the loss function MSE and the activating function Log-sigmoid can be selected to be suitable for training and classification of skin diseases effectively.

In the MLNN with back-propagation, mean square error (MSE) is the most commonly used regression loss function. The MSE is the sum of squared distances between the target variable and predicted values and calculated,

$$
M S E=\frac{1}{n} \sum_{i=1}^{n}\left(y_{i}-\hat{y}_{i}\right)^{2}
$$

where $y_{i}$ is the desired neural network output, and $\hat{y}_{i}$ is the neural network output and $\mathrm{n}$ is the number of output nodes. In addition, in this MLNN, logsig is a transfer function for calculating a layer's the output $y$ from its net input $\mathrm{x}$ : 


$$
y(x)=\frac{1}{1+e^{-x}}
$$

In this MLNN, the input dataset is a vector of six optimal features extracted from the GLCM algorithm. In addition, each skin disease in five types is constructed with a set of 100 images, which would greatly enhance the classification accuracy.

\section{EXPERIMENTAL RESULTS}

\subsection{Skin disease datasets}

Datasets, in this study, are from ISIC database, including 10,000 images for seven types of skin diseases. For the evaluation of classification effectiveness, 500 images of skin diseases were chosen to be 100 images for each type, in which each dataset was divided 80 images for training and 20 ones for testing as described in Table 1.

Table 1. Representation of datasets for training and testing of five types of skin disease

\begin{tabular}{cccccc}
\hline Training and testing images & Basal cell carcinoma & Benign keratosis & Dermatofibroma & Melanocytic nevus & Melanoma \\
\hline Training & 100 & 100 & 100 & 100 & 100 \\
Testing & 20 & 20 & 20 & 20 & 20
\end{tabular}

\subsection{Separation of ROI}

In this article, the skin disease images were resized to the $512 \times 512$ same size and then labeled as shown in Figure 1. Before separating ROI from a skin disease image, image processing methods were applied. In particular, the image with the ROI was enhanced using the kernel $K_{1}$ in (1) as described in Figure 2. In addition, unnecessary noises in the image were removed using the Butterworth high-pass filter in the frequency domain as shown in Figure 3. This image was converted to the binary image with the black ROI and the white background as shown in Figure 4. Next, the binary image was processed to continuously eliminate unnecessary details using the median filter as shown in Figure 5, then it removed skin hair detail using the bottom-hat filter with kernel $\mathrm{K}_{2}$ in (3) as described in Figure 6. The erosion algorithm was applied to fill the skin disease areas as shown in Figure 7.

$$
\mathbf{K}_{2}=\left[\begin{array}{lllllll}
0 & 0 & 1 & 1 & 1 & 0 & 0 \\
0 & 1 & 1 & 1 & 1 & 1 & 0 \\
1 & 1 & 1 & 1 & 1 & 1 & 1 \\
1 & 1 & 1 & 1 & 1 & 1 & 1 \\
1 & 1 & 1 & 1 & 1 & 1 & 1 \\
0 & 1 & 1 & 1 & 1 & 1 & 0 \\
0 & 0 & 1 & 1 & 1 & 0 & 0
\end{array}\right]
$$

For the ROI separation, the Canny edge detection was utilized in the binary image as shown in Figure 8. The erosion method was applied for linking edges of objects as shown in Figure 9, then they were filled all object regions and labeled as shown in Figure 10. The ROI area in filled binary image was extracted based on the largest ROI area as shown in Figure 11, then it was multiplied to the enhanced original image to produce the enhanced original ROI as shown in Figure 12 before extracting features for classification of skin diseases.

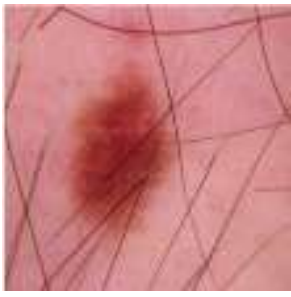

Figure 1. Image resized $512 \times 512$

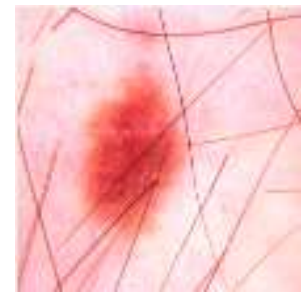

Figure 2. Image after enhancement

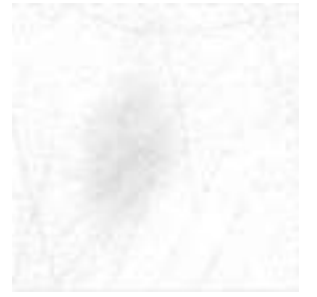

Figure 3. Image after butterworth high-pass

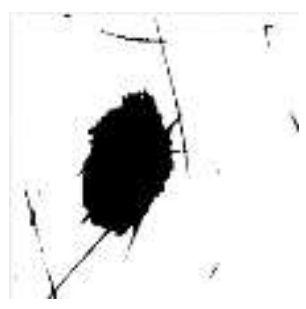

Figure 4. Binary image 


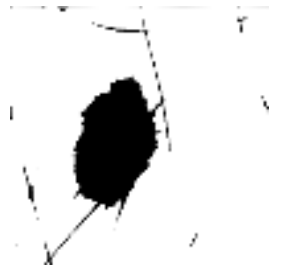

Figure 5. Image after the median filter

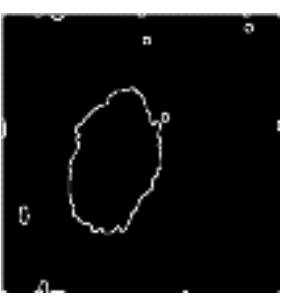

Figure 9. Edge image eroded

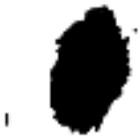

Figure 6. Image eliminated skin hairs

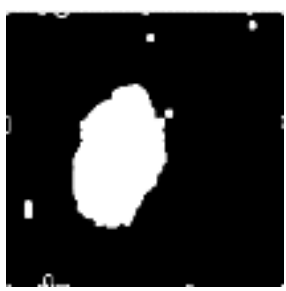

Figure 10. Image with the filled objects

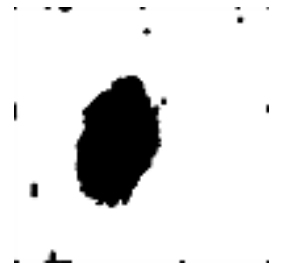

Figure 7. Image after the erosion

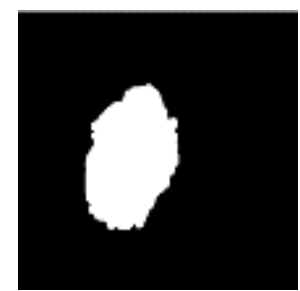

Figure 11. Image with the largest area

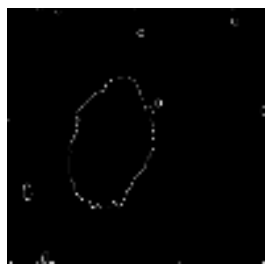

Figure 8. Image with the Canny edge detection

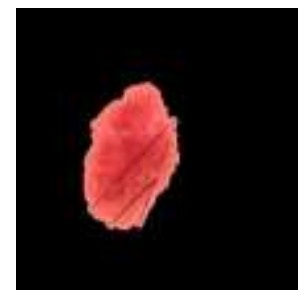

Figure 12. Original ROI image

All skin disease images have been processed using image processing methods. Figure 13 showed ROI images separated from the processed image of 5 types of skin diseases such as basal cell carcinoma (Figure a0-a1), benign keratosis (Figure b0-b1), dermatofibroma (Figure c0-c1), melanocytic nevus (Figure d0-d1), melanoma (Figure e0-e1). The ROI image retains most of the diseased skin area and unnecessary areas are removed for classification. The accurate ROI separation is important, because the amount of the important feature information in the ROI makes it calculate features faster and more accurate for classification. Therefore, features in the ROI images were extracted using the GLCM method, in which 6 optimal features of 11 ones were selected for training and classify skin diseases. With the original ROIs separated from 5 skin diseases, it is obvious that there is the structural difference among shapes, colors and others. From these different factors, 6 opitmal features extracted using the GLCM possibly enhance the classification accuracy.

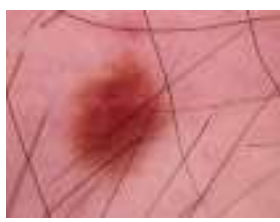

(a0)

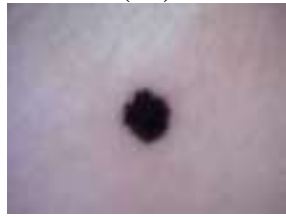

(c0)

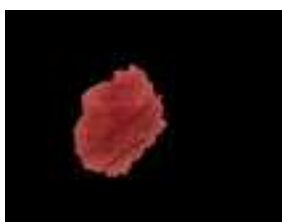

(a1)

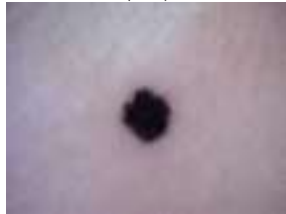

(c1)

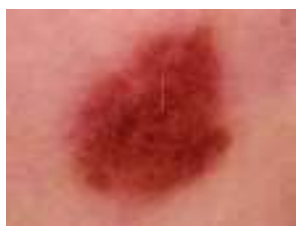

(b0)

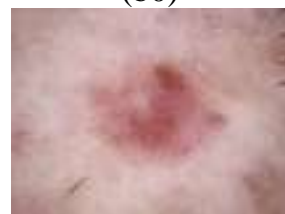

(d0)

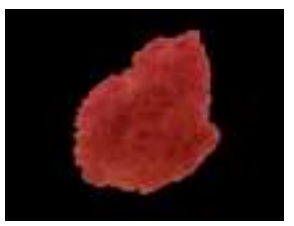

(b1)

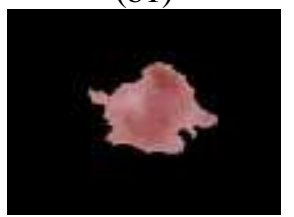

(d1)

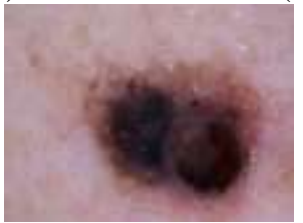

(e0)

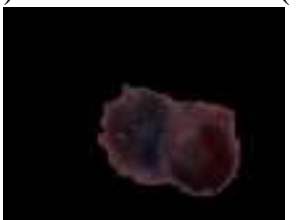

(e1)

Figure 13. Representation of the original and the ROI images separated after enhancement and segentation;

(a0) basal cell carci-noma, (a1) ROI of basal cell carci-noma image, (b0) benign keratosis, (b1) ROI of

benign keratosis image, (c0) dermato-fibroma, (c1) ROI of dermato-fibroma image, (d0) melano-cytic nevus, (d1) ROI of melano-cytic nevus image, (e0) melano-ma, and (e1) ROI of melano-ma image 


\subsection{Feature extraction from ROIs using a GLCM algorithm}

Figure 14 showed the values of 11 features calculated using the GLCM algorithm, in which 10 images of Basal cell carcinoma (cell carcinoma) were used. Based on information in Figure 14, we can easily evaluate that each of 11 features for the 10 skin disease images is nearly similar. This is the basis of using the GLMC algorithm for extracting features.

Table 2 and Table 3 showed the Min-Max threshold values of each feature in five types of skin diseases, including: basal cell carcinoma (No. 1), benign keratosis (No. 2), dermatofibroma (No. 3), melanocytic nevus (number 4), melanoma (No. 5). In addition, from Table 2, two features of smoothness and IDM have the Min (1.0) and Max (1.0) values corresponding to each disease without change, respectively, so it was not selected. In addition to two Smoothness and IDM features, correlation feature has the too small difference between Min and Max values, just 0.05, it was not chosen. While two feature pairs of mean-variance and RMS-contrast are similar, so we just choose one pair of mean and contrast. The group of six features selected including: contrast, energy, homogeneity, mean, standard deviation, and entropy differ greatly when comparing the min and max values for diseases and can be used for training in the classifier.

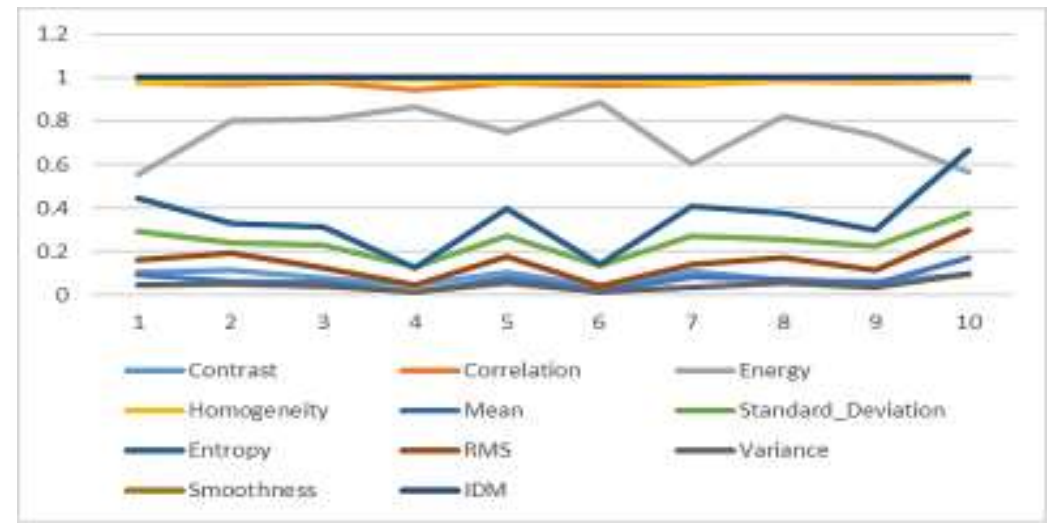

Figure 14. Representation of 11 features of 10 Basal cell carcinoma images

Table 2. Eleven average features extracted from five types of skin disease using the GLCM

\begin{tabular}{ccccccccccccc}
\hline Skin disease class & \multicolumn{2}{c}{ Contrast } & \multicolumn{2}{c}{ Homogeneity } & \multicolumn{2}{c}{ Entropy } & \multicolumn{3}{c}{ Mean } & \multicolumn{3}{c}{ RMS } \\
& $\min$ & $\max$ & $\min$ & $\max$ & $\min$ & $\max$ & $\min$ & $\max$ & $\min$ & $\max$ & $\min$ & $\max$ \\
\hline 1 & 0.037 & 0.211 & 0.958 & 0.995 & 0.127 & 0.733 & 0.018 & 0.205 & 0.042 & 0.374 & 0.346 & 0.939 \\
2 & 0.058 & 0.825 & 0.946 & 0.993 & 0.238 & 0.978 & 0.039 & 0.414 & 0.096 & 0.552 & 0.335 & 0.825 \\
3 & 0.022 & 0.158 & 0.969 & 0.998 & 0.033 & 0.355 & 0.003 & 0.067 & 0.022 & 0.153 & 0.463 & 0.974 \\
4 & 0.036 & 0.324 & 0.953 & 0.997 & 0.130 & 0.973 & 0.018 & 0.997 & 0.052 & 0.563 & 0.334 & 0.947 \\
5 & 0.036 & 0.420 & 0.915 & 0.995 & 0.076 & 0.806 & 0.009 & 0.247 & 0.050 & 0.417 & 0.252 & 0.941 \\
\hline
\end{tabular}

Table 3. Eleven average features extracted from five types of skin disease using the GLCM

\begin{tabular}{ccccccccccc}
\hline Skin disease class & \multicolumn{2}{c}{ Standard Deviation } & \multicolumn{2}{c}{ Varience } & \multicolumn{2}{c}{ Smoothness } & \multicolumn{2}{c}{ Correlation } & \multicolumn{3}{c}{ IDM } \\
& $\min$ & $\max$ & $\min$ & $\max$ & $\min$ & $\max$ & $\min$ & $\max$ & $\min$ & $\max$ \\
\hline 1 & 0.131 & 0.404 & 0.014 & 0.123 & 1.000 & 1.000 & 0.938 & 0.988 & 1.000 & 1.000 \\
2 & 0.194 & 0.493 & 0.022 & 0.160 & 1.000 & 1.000 & 0.965 & 0.989 & 1.000 & 1.000 \\
3 & 0.059 & 0.250 & 0.003 & 0.046 & 1.000 & 1.000 & 0.861 & 0.973 & 1.000 & 1.000 \\
4 & 0.133 & 0.491 & 0.015 & 0.177 & 1.000 & 1.000 & 0.953 & 0.989 & 1.000 & 1.000 \\
5 & 0.096 & 0.431 & 0.009 & 0.141 & 1.000 & 1.000 & 0.835 & 0.984 & 1.000 & 1.000 \\
\hline
\end{tabular}

Figure 15 represented six feature statistics for five image datasets of skin diseases to illustrate the difference. This is the basis for choosing these six features for training in the classifier. In particular, Figure 15(a) presented the contrast parameters of five classes of skin diseases averaged from 100 images for each class. It is similar to calculation of the remaining features such as the entropy values as shown in Figure 15(b); the uniformity value as shown in Figure 15(c); the value of energy as shown in Figure 15(d); the mean value as shown in Figure 15(e); and standard deviation value as shown in Figure 15(f). From Figure 15, it can be seen that the class of dermatofibroma (No. 3) has the lowest feature values, including contrast, entropy, mean and standard deviation, while the two features of homogeneity and energy are the highest at 0.996 and 0.926, respectively. In addition, from the data in Figure 15, melanocytic nevus (No. 4) has the 2nd 
highest feature values of all five diseases, followed by basal cell carcinoma disease (No. 1). Through the analysis of the mean values of the skin disease features, it can be seen that the difference between five disease image datasets is quite clear for applying to disease classification.

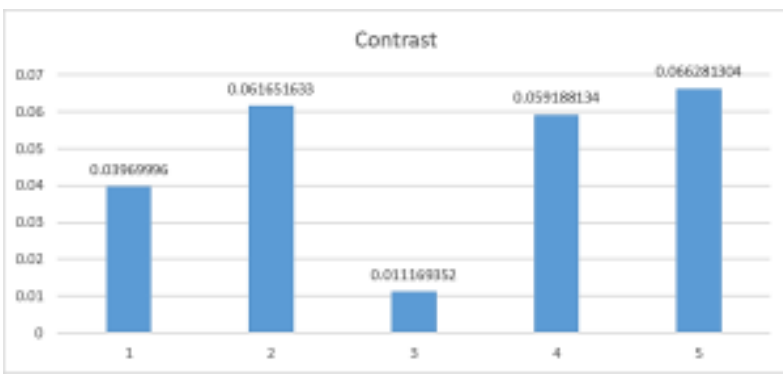

(a)

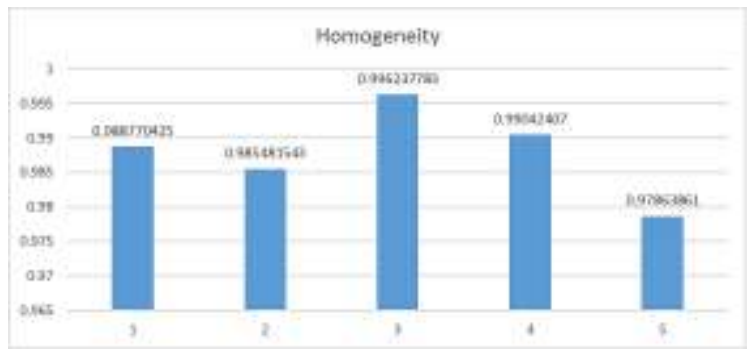

(c)

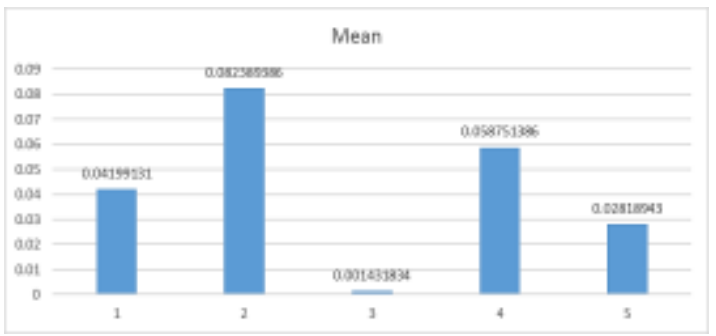

(e)

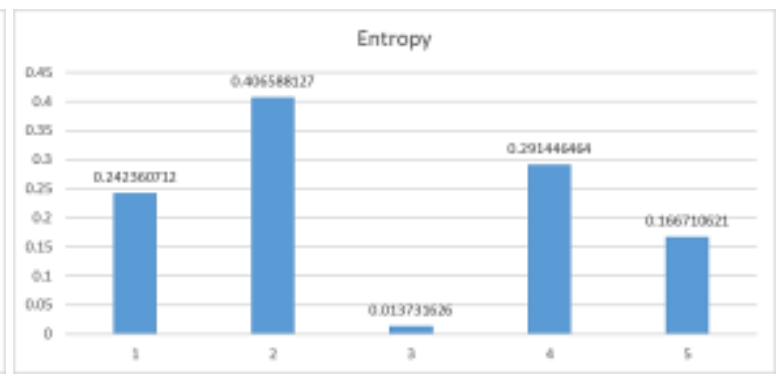

(b)

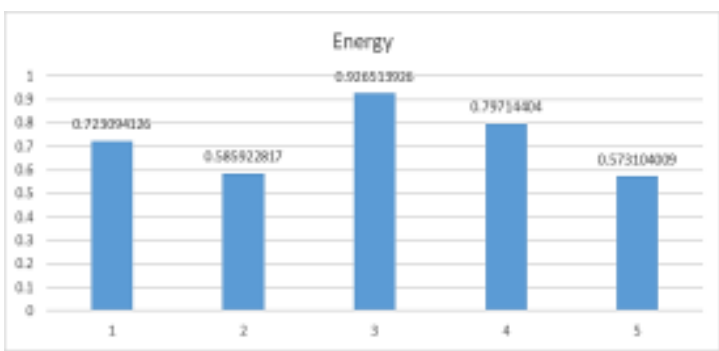

(d)

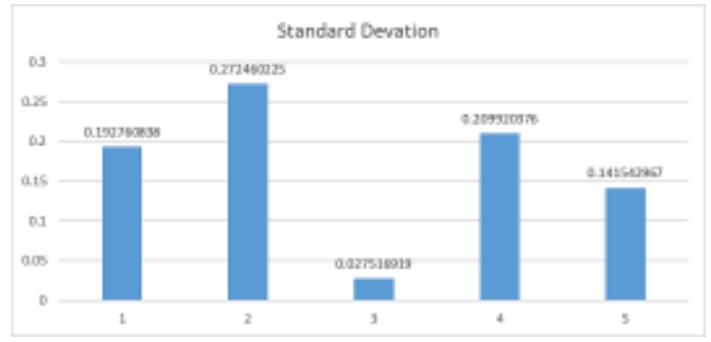

(f)

Figure 15. Representation of the average statistics of 6 features of 5 skin disease datasets; (a) representation of contrast, (b). representation of entropy, (c). representation of homogeneity, (d). representation of energy, (e). representation of mean, and (f). representation of standard deviation

\subsection{Classification accuracy using a MLNN structure}

The MLNNs model employed in this study includes an input layer with 6 nodes corresponding to 6 feature vectors; 3 hidden layers with 100 nodes for each layer; and an output layer with 5 nodes corresponding 5 classes of skin disease needed for classification as shown in Figure 16. In addition, more or less hidden layers could be chosen to possibly ensure the best classification. The MLNN was employed to perform training with the learning speed of 10-4 with unchange during the learning process, the desired model error was 7.10-3. Figure 17 showed the training error curve, in which the error of the model continuously decreased following the curve and it achieved the best value of 0.0068 after 449 epochs. This shows that the model achieved convergence with fast training time.

After training 400 images of 5 classes, the MLNN was applied to classify skin diseases. Classification results were tested on 100 images corresponding to 5 disease classes. To evaluate the classification accuracy, a confusion matrix in Figure 18 was employed to show the average classification accuracy of $92 \%$, in which the accuracy of the skin diseases is $85 \%$ basal cell carcinoma (No. 1), 95\% Benign keratosis (No. 2), 100\% dermatofibroma (No. 3), 85\% melanocytic nevus (No. 4), 95\% melanoma (No. 5), respectively. In the classification result of diseases, dermato-fibroma disease has the highest accuracy of $95 \%$ due to its feature being very different compared to 4 remaining diseases. While basal cell carcinoma and melanocytic nevus diseases have the lowest accuracies of $85 \%$. In the case of basal cell carcinoma classification, the minor error classification is due to its feature mainly confused with that of 
benign keratosis. In particular, when classifying melanocytic nevus disease, 2 of 20 images (10\% of the total number) is error due to confusing with Basal cell carcinoma disease.

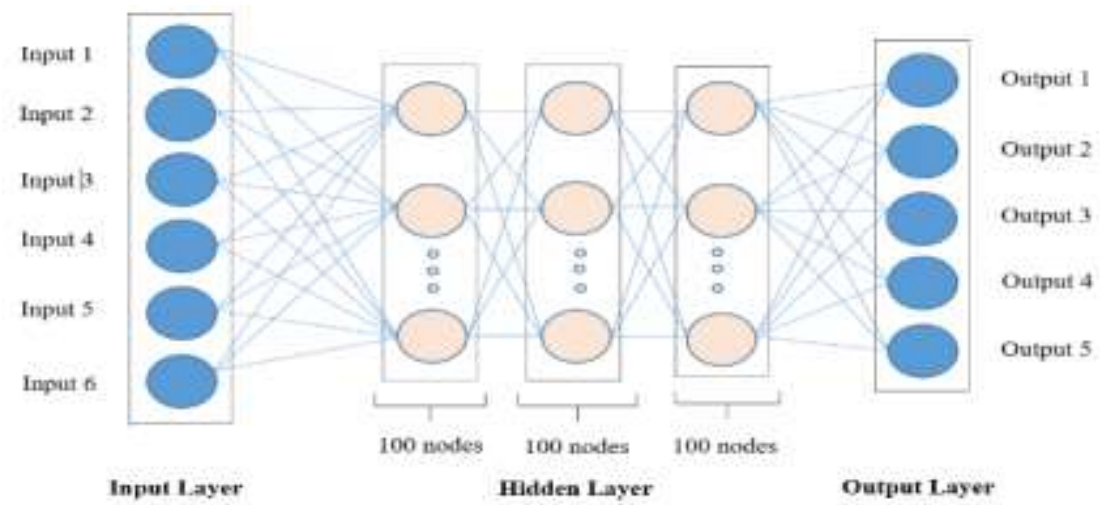

Figure 16. Classification model of the MLNN structure for 6 input features and 5 output classes

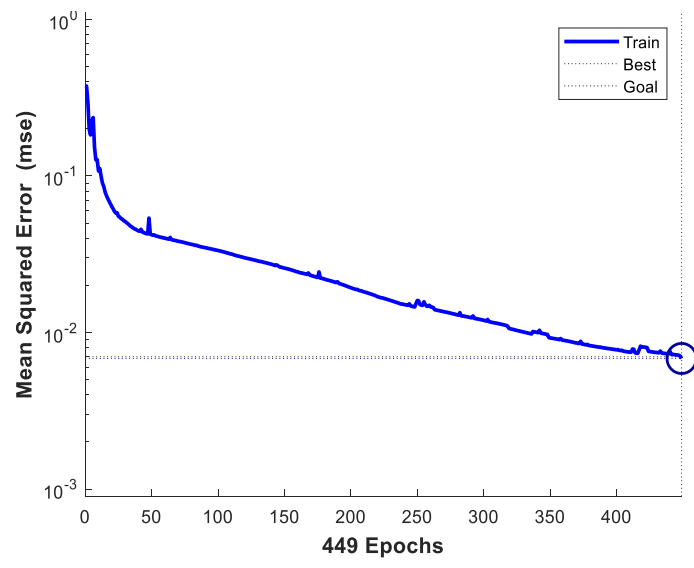

Figure 17. Training result using the MLNN structure

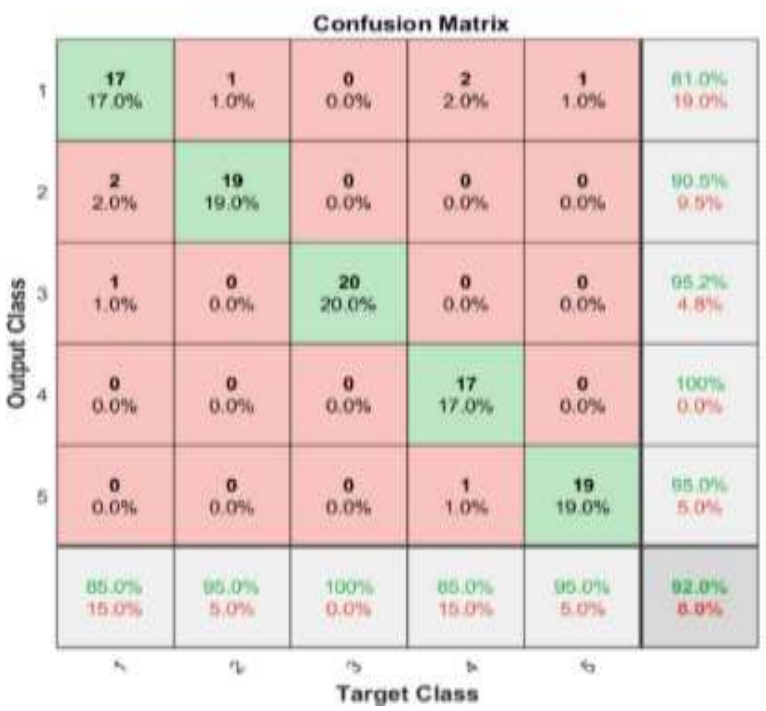

Figure. 18. Confusion matrix for evaluation of 20 testing images each class

Table 4 presented the comparison of the accurate results classifying 5 classes of skin diseases based on 3 groups of different features. The average results showed that the training model using only 6 features (contrast, energy, homogeneity, mean, standard deviation, entropy) has the $92 \%$ highest accuracy; the lowest accuracy of $71 \%$ using 11 features, and using only 3 features producing $78 \%$. It is obvious that the selected group of 6 features using the GLCM represents the effectiveness of classifying the five skin diseases.

Table 5 showed that the result using the proposed method has the $92 \%$ classification accuracy for 5 classes of skin diseases, it is $2 \%$ higher than the best method [38] of the previous researches and about $6 \%$ higher than the lowest accuracy [34]. With the high classification accuracy thanks to image processing to extract the ROI with appropriate methods, in which almost information is possibly kept in the ROI. Moreover, the GLCM has been applied for many previous studies [38] and results has shown very positive. However, in this study, we chose only 6 features of 11 features that can highly contain a lot of important information related to skin disease. In addition, the 6 features applied for training to be able to condense and less time, so it can increase the classification accuracy.

In addition to the selection of image processing methods and the selection of 6 features, Table 5 showed that the MLNN was proposed for the appropriate number of nodes and layers to achieve a result with higher accuracy compared to other models as CNN [31], FRCNN [34], Depthwise separable CNN [32] and SVM [38]. In particular, authors represented combining the GLCM and the SVM for classifying 3 classes of skin diseases and achieved the accuracy of $90 \%$. In addition, our proposed method here has less training time 
compared to $\mathrm{CNN}$ or previous research methods. In particular, with our proposed model, it only took 25 minutes to separate ROIs and extract features from 500 images, 10 seconds to train these feature data, compared with a training time of 25 minutes, 90 minutes, 70 minutes, and 230 minutes for using the AlexNet, VGG16, ResNet-18 and ResNet-101 models with the ISIC dataset [31]. It means that the training time of the proposed model is small because the MLNN is simpler with fewer parameters. From the results obtained, we conclude that our model is able to efficiently extract the features and then produces better results with the very high accuracy.

Table 4. Comparison of the classification accuracy of 3 groups from 5 skin disease datasets

\begin{tabular}{lccccc}
\hline \multicolumn{1}{c}{ Feature groups } & $\begin{array}{c}\text { Average } \\
\text { Accuracy }\end{array}$ & $\begin{array}{c}\text { Basal cell } \\
\text { carcinoma }\end{array}$ & $\begin{array}{c}\text { Benign } \\
\text { keratosis }\end{array}$ & $\begin{array}{c}\text { Dermatofibroma } \\
\text { Melanocytic } \\
\text { nevus }\end{array}$ & $\begin{array}{c}\text { Melanoma } \\
\text { Contrast, Energy, Entropy }\end{array}$ \\
$\begin{array}{l}\text { Contrast, Energy, Homogeneity, } \\
\text { Mean, Standard Deviation, Entropy }\end{array}$ & $92 \%$ & $75 \%$ & $75 \%$ & $75 \%$ & $65 \%$ \\
All features & $71 \%$ & $85 \%$ & $95 \%$ & $100 \%$ & $85 \%$ \\
\hline
\end{tabular}

Table 5. Comparison of previous methods with parameters and our proposed method

\begin{tabular}{|c|c|c|c|c|c|}
\hline Method & Pre-Processing Technique & Image set & Architecture & Accuracy $(\%)$ & Types \\
\hline Mahbod [31] & $\begin{array}{l}\text { Colour standardisation, } \\
\text { normalisation, Resizing }\end{array}$ & $\begin{array}{l}\text { Malignant melanoma: } 441 \\
\text { Seborrheic keratosis:296 } \\
\text { Benign nevi: } 1450\end{array}$ & $\mathrm{CNN}$ & 87.7 & 3 \\
\hline Shunichi [34] & Non & $\begin{array}{l}\text { Malignant melanoma: } 1611 \\
\text { Basal cell carcinoma: } 401 \\
\text { Nevus: } 2837 \\
\text { Seborrheic keratosis: } 746 \\
\text { Senile lentigo: } 79 \\
\text { Hematoma/Hemangioma: } 172\end{array}$ & FRCNN & 86.2 & 6 \\
\hline Sara [32] & $\begin{array}{l}\text { Image normalization, data } \\
\text { standardization }\end{array}$ & $\begin{array}{l}\text { Melanoma: } 1113 \\
\text { Melanocytic nevus: } 6705 \\
\text { Basal cell carcinoma: } 514 \\
\text { Vascular lesion: } 142 \\
\text { Actinic keratosis: } 327 \\
\text { Benign keratosis: } 1099 \\
\text { Dermatofibroma: } 115\end{array}$ & $\begin{array}{l}\text { Depthwise } \\
\text { separable } \\
\text { CNN }\end{array}$ & 87.24 & 6 \\
\hline $\begin{array}{l}\text { Li-sheng Wei } \\
\text { [38] }\end{array}$ & Image segmentation & $\begin{array}{l}\text { Herpes : } 30 \\
\text { Dermatitis: } 30 \\
\text { Psoriasis: } 30\end{array}$ & $\begin{array}{c}\text { GLCM + } \\
\text { SVM }\end{array}$ & 90 & 3 \\
\hline $\begin{array}{l}\text { Proposed } \\
\text { model }\end{array}$ & ROI separation & $\begin{array}{l}\text { Basal cell carcinoma: } 100 \\
\text { Benign keratosis: } 100 \\
\text { Dermatofibroma: } 100 \\
\text { Melanocytic nevus: } 100 \\
\text { Melanoma: } 100\end{array}$ & $\begin{array}{c}\text { GLCM + } \\
\text { MLNN }\end{array}$ & $92 \%$ & 5 \\
\hline
\end{tabular}

\section{CONCLUSION}

In this study, we have presented the high accurate classification of five types of skin diseases. In particular, filter, segmentation, and separation of the best ROI images from skin disease images were applied for extraction of six optimal features using the GLCM. It is obvious that focusing on processing image for extracting the optimal features saved time of training using the MLNN. In addition, we selected 100 images for each skin disease type and thus the balance of the image datasets increases the classification performance. From the datasets, the MLNN with one 6-nodes input layer, one 5-nodes output layer and 3 hidden layers with 100 nodes for each layer was applied and produced the high classification accuracy for the group of the 5-types skin diseases compared to other groups in Table 3. Moreover, the accurate classification results were evaluated using the matrix confusion and this showed to illustrate the effectiveness of the proposed classification method. Therefore, this classification method can provide a sophisticated way to classify complex data with higher accuracy. In addition, it can be improved by using much larger and diverse datasets for training in the neural network on a much larger and diverse dataset with high intra-class variability due to this would decrease the misclassification.

\section{ACKNOWLEDGEMENTS}

This work is supported by Ho Chi Minh City University of Technology and Education (HCMUTE) under Grant No. T2020-29TD. 


\section{REFERENCES}

[1] Chante Karimkhani, et al, "Global Skin Disease Morbidity and Mortality An Update From the Global Burden of Disease Study 2013," JAMA Dermatology | Original Investigation, vol. 153, no. 5, pp. 406-412, 2017, doi: 10.1001/jamadermatol.2016.5538.

[2] Thanh-Hai Nguyen, "Wavelet-based Image Fusion for Enhancement of ROI in CT Image," Journal of Biomedical Engineering and Medical Imaging, vol. 1, no. 4, pp. 1-13, 2014, doi:10.14738/jbemi.14.310.

[3] K. Melbin and Y. J. V. Raj, "An Enhanced Model for Skin Disease Detection using Dragonfly Optimization based Deep Neural Network," Third International conference on I-SMAC, pp. 346-351, 2019, doi: 10.1109/ISMAC47947.2019.9032458

[4] N. Mittal, S. Tanwar and S. K. Khatri, "Identification \& enhancement of different skin lesion images by segmentation techniques," the 6th International Conference on Reliability, Infocom Technologies and Optimization (ICRITO), pp. 609-614, 2017, doi: 10.1109/ICRITO.2017.8342500.

[5] Dogra A and Bhalla P, "Image Sharpening By Gaussian And Butterworth High Pass Filter," Biomedical \& Pharmacology Journal, vol. 7, no. 2, pp. 707-713, 2014, doi: 10.13005/bpj/545.

[6] Bharti Lalotra, Renu Vig and Sumit Budhiraja, "Multimodal medical image fusion using Butterworth high pass filter and Cross bilateral filter," 4th International Conference on Advancements in Engineering \& Technology (ICAET), pp. 1021-1026, 2016, doi: 10.1051/matecconf/20165701021.

[7] Do Hyun Chung and G. Sapiro, "Segmenting skin lesions with partial differential-equations-based image processing algorithms," IEEE Transactions on Medicine Image, vol. 19, no. 7, pp. 763-767, 2000, doi: $10.1109 / 42.875204$.

[8] R. Sumithra, Mahamad Suhil and D.S. Guru, "Segmentation and classification of skin lesions for disease diagnosis," Procedia Computer Science, vol. 45, pp. 76-85, 2015, doi: 10.1016/j.procs.2015.03.090.

[9] Abbas F. H. Alharan, Hayder K. Fatlawi and Nabeel Salih Ali, "A cluster-based feature selection method for image texture classification," Indonesian Journal of Electrical Engineering and Computer Science, vol. 14, no. 3, pp. 1433-1442, 2019, doi: 10.11591/ijeecs.v14.i3.pp1433-1442.

[10] H. J. Abd, Ahmad S. Abdullah and Muhammed Salah Sadiq Alkafaji, "A new swarm intelligence information technique for improving information balancedness on the skin lesions segmentation," International Journal of Electrical and Computer Engineering (IJECE), vol. 10, no. 6, pp. 5703-5708, 2020, doi: 10.11591/ijece.v10i6.pp5703-5708.

[11] Cam Q. T. Thanh and Nguyen T. Hai, "Trilinear Interpolation Algorithm for Reconstruction of 3D MRI Brain Image," American Journal of Signal Processing, vol. 7, no. 1, pp. 1-11, 2017, doi:10.5923/j.ajsp.20170701.01.

[12] J. Lu, E. Kazmierczak, J. H. Manton and R. Sinclair, "Automatic segmentation of scaling in 2-D psoriasis skin images," IEEE Transaction on Medical Imaging, vol. 32, no. 4, pp. 719-730, 2013, doi: 10.1109/TMI.2012.2236349.

[13] P. N. H. Tra, N. T. Hai and T. T. Mai, "Image Segmentation for Detection of Benign and Malignant Tumors," Proceedings of IEEE 3rd International Conference on Biomedical Engineering, pp. 51-54, 2016, doi: 10.1109/BME-HUST.2016.7782105.

[14] Anaswara S. Mohan and Seena S. Das, "Medical Image Enhancement Techniques by Bottom Hat and Median Filtering," International Journal of Electronics Communication and Computer Engineering, vol. 5, no. 4, pp. 347-351, 2014, doi:10.4236/jdaip.2016.41001.

[15] Jaseema Yasmin and Mohamed Sathik, "An Improved Iterative Segmentation Algorithm using Canny Edge Detector with Iterative Median Filter for Skin Lesion Border Detection," International Journal of Computer Applications, vol 50, no. 6, pp. 37-42, 2012, doi:10.5120/7779-0865.

[16] R Zeinali, A Keshtkar, A Zamani and N Gharehaghaji, "Brain Volume Estimation Enhancement by Morphological Image Processing Tools," Journal of Biomedical Physics and Engineering, vol. 7, no. 4, pp. 379-388, 2017, doi:10.22086/jbpe.v0i0.586.

[17] S. Cerrato, L. Ramió-Lluch, D. Fondevila, D. Rodes, P. Brazis, and A. Puigdemont, "Effects of Essential Oils and Polyunsaturated Fatty Acids on Canine Skin Equivalents: Skin Lipid Assessment and Morphological Evaluation," Journal of Veterinary Medicine, vol. 2013, pp. 1-9, 2013, doi: 10.1155/2013/231526.

[18] Tatyana Polevaya, I.A. Saitov, Roman Ravodin and Andrey Filchenkov, "Image classification of primary morphological elements of skin lesions," Scientific and Technical Journal of Information Technologies Mechanics and Optics, vol. 19, no. 2, pp. 333-338, 2019, doi:10.17586/2226-1494-2019-19-2-333-338.

[19] M. Abdullah-Al-Wadud and O. Chae, "Region-of-Interest Selection for Skin Detection Based Applications," International Conference on Convergence Information Technology (ICCIT 2007), pp. 1999-2004, 2007, doi: 10.1109/ICCIT.2007.267.

[20] Pedro M. M. Pereira, et al, "Dermoscopic skin lesion image segmentation based on Local Binary Pattern Clustering: Comparative study," Biomedical Signal Processing and Control, vol. 59, pp. 1-12, 2020, doi: 10.1016/j.bspc.2020.101924.

[21] Qiaohua Liu, et al, "Research on Skin Texture Classification by Gray Level Co-occurrence Matrix and the BP Neural Network," International Conference on Test, Measurement and Computational Method (TMCM), pp. 26-29, 2015, doi: 10.2991/tmcm-15.2015.8.

[22] Shervan Fekri Ershad, "Texture Classification Approach Based on Combination of Edge \& Co-occurrence and Local Binary Pattern," International Conference on Computer Vision and Pattern Recognition, pp. 626-629, 2011.

[23] Suhair H S Al-Kilidar and Loay E. George, "Texture Recognition Using Co-occurrence Matrix Features and Neural Network," Journal of Theoretical and Applied Information Technology, vol. 95, no. 21, pp. 5949-5961, 2017. 
[24] Z. F. Jabr and M. A. A. Hasan, "Diagnosing of some hepatic lesions from light microscope images based on morphological and texture features," Indonesian Journal of Electrical Engineering and Computer Science (IJEECS), vol. 18, no. 2, pp. 995-1003, 2020, doi: 10.11591/ijeecs.v18.i2.pp995-1003.

[25] S. Arivazhagan, R.Newlin Shebiah, K. Divya and M. P. Subadevi, "Skin disease classification by extracting independent components," Journal of Emerging Trends in Computing and Information Sciences, vol. 3, no. 10, pp. 1379-1382, 2012.

[26] Lakshay Bajaj, Himanshu Kumar and Yasha Hasija, "Automated System for Prediction of Skin Disease using Image Processing and Machine Learning," International Journal of Computer Applications, vol. 180, no. 19, pp. 9-12, 2018, doi: 10.5120/ijca2018916428.

[27] D. A. Gavrilov, A. V. Melerzanov, N. N. Shchelkunov and E. I. Zakirov, "Use of Neural Network-Based Deep Learning Techniques for the Diagnostics of Skin Diseases," Biomedical Engineering, vol. 52, pp. 348-352, 2019, doi: 10.1007/s10527-019-09845-9.

[28] Danilo Barros Mendes and Nilton Correia da Silva, "Skin Lesions Classification Using Convolutional Neural Networks in Clinical Images," Computer Vision and Pattern Recognition, pp. 1-11, 2018.

[29] Andre Esteva, Brett Kuprel and Sebastian Thrun, "Deep Networks for Early Stage Skin Disease and Skin Cancer Classification," Stanford University, 2015.

[30] Ammara Masood, Adel Ali Al-Jumaily and Tariq Adnan, "Development of Automated Diagnostic System for Skin Cancer: Performance Analysis of Neural Network Learning Algorithms for Classification," in Wermter S. et al. (eds) Artificial Neural Networks and Machine Learning - ICANN 2014. Lecture Notes in Computer Science, vol. 8681, pp. 837-844, 2014, doi: 10.1007/978-3-319-11179-7_105.

[31] Mahbod A, Amirreza Mahbod, Gerald Schaefer, Isabella Ellinger, Rupert Ecker, Alain Pitiot, Chunliang Wang, "Fusing fine-tuned deep features for skin lesion classification," Computerized medical imaging and graphics, vol. 71, pp. 19-29, 2019, doi: 10.1016/j.compmedimag.2018.10.007.

[32] S. H. Kassani, P. H. Kassani, M. J. Wesolowski, K. A. Schneider and R. Deters, "Depthwise Separable Convolutional Neural Network for Skin Lesion Classification," IEEE International Symposium on Signal Processing and Information Technology (ISSPIT), pp. 1-6, 2019, doi: 10.1109/ISSPIT47144.2019.9001790.

[33] Muhammad Naseer Bajwa, et al, "Computer-Aided Diagnosis of Skin Diseases using Deep Neural Networks," Applied Sciences, vol. 10, no. 7, pp. 1-13, 2020, doi: 10.3390/app10072488.

[34] Shunichi Jinnai, Naoya Yamazaki, Yuichiro Hirano, Yohei Sugawara, Yuichiro Ohe, and Ryuji Hamamoto, "The Development of a Skin Cancer Classification System for Pigmented Skin Lesions Using Deep Learning," Biomolecules, vol. 10, no. 8, pp. 1123-1135, 2020, doi: 10.3390/biom10081123.

[35] Rosniza Roslan, Iman Najwa Mohd Razly, Nurbaity Sabri and Zaidah Ibrahim, "Evaluation of psoriasis skin disease classification using convolutional neural network", IAES International Journal of Artificial Intelligence (IJ-AI), vol. 9, no. 2, pp. 349-355, 2020, doi: 10.11591/ijai.v9.i2.pp349-355.

[36] Amiri S, Movahedi MM, Kazemi K and Parsaei H, "An Automated MR Image Segmentation System Using Multi-layer Perceptron Neural Network," Journal of biomedical physics \& engineering, vol. 3, no. 4, pp. 115-22, 2013.

[37] Sugiarti, Yuhandri, Na'am Jufriadif, Indra Dolly and Santony Julius, "An artificial neural network approach for detecting skin cancer," TELKOMNIKA, vol. 17, no. 2, pp. 788-793, 2019, doi: 10.12918/TELKOMNIKA.v17i29547.

[38] Li-sheng Wei, Quan Gan, Tao Ji, "Skin Disease Recognition Method Based on Image Color and Texture Features," Computational and Mathematical Methods in Medicine, vol. 2018, pp. 1-10, 2018, doi: 10.1155/2018/8145713.

\section{BIOGRAPHIES OF AUTHORS}

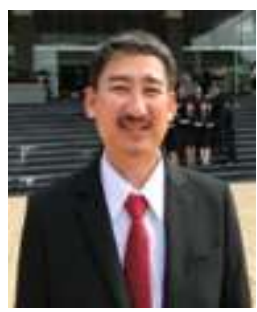

Thanh-Hai Nguyen received his BEng. degree with Electronics engineering from the HCMC University of Technology and Education, in Vietnam, 1995; MEng. One with Telecommunication and Electronics Engineering from HCMC University of Technology (UTE), in Vietnam, 2002; PhD. degree with Electronics Engineering from University of Technology, Sydney in Australia, 2010. Currently, he is a lecturer in the Department of Industrial Electronic - Biomedical Engineering, Faculty of Electrical - Electronics Engineering, the HCMCUTE, Vietnam. His research interests are Bio-signal and image processing, machine learning, smart wheelchairs and Artificial intelligence. (Email: nthai@hcmute.edu.vn).

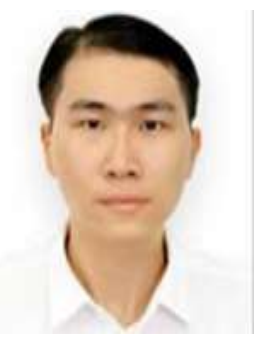

Ba-Viet Ngo received his M.Eng. in Electronics Engineering from HCMC University of Technology and Education in 2014. He is a PhD. student in Electronics Engineering at HCM City University of Technology and Education. His research interests include smart wheelchair, artificial intelligence, image processing. (Email: vietnb@hcmute.edu.vn). 